La révision de la loi sur les stupéfiants a été le thème central de la rencontre organisée le $1^{\text {er }}$ octobre 2008 par la FMH à l'intention des parlementaires. Ceux-ci ont été particulièrement nombreux à répondre à notre invitation et à venir discuter de ce projet de politique de santé qui sera soumis au vote populaire à fin novembre. Le point culminant de cette rencontre fut l'exposé du Dr Ambros
Uchtenhagen, qui expliqua clairement pourquoi on ne peut que soutenir la loi révisée sur les stupéfiants. Le Comité central de la FMH de même que les délégués ont déjà approuvé ce projet il y a quelques semaines.

Dr Jacques de Haller, président de la FMH

\title{
Echouer à son propre succès? Quelques bonnes raisons pour accepter la révision de la LStup
}

\section{Ambros Uchtenhagen}

Président de l'Institut de recherche sur la santé publique et les addictions, associé à l'université de Zurich

Correspondance:

Prof. Dr. méd. et phil.

Ambros Uchtenhagen

Institut de recherche sur la santé

publique et les addictions

Konradstrasse 8

CH-8031 Zurich

Tél. 0444481160

Fax 0444481170

isgf@isgf.uzh.ch

www.isgf.ch
La révision de la loi sur les stupéfiants (LStup), telle qu'elle est proposée, est convaincante car elle permettra de poursuivre la politique actuelle des quatre piliers (prévention, thérapie, réduction des risques, répression), d'avoir la base légale nécessaire pour agir au niveau fédéral, et de poursuivre le traitement des personnes gravement toxicodépendantes avec la prescription d'héroïne.

Avant l'instauration de cette politique, la situation était catastrophique sur le front de la drogue: malgré la répression, les scènes ouvertes de la drogue et leur misère omniprésente avaient pris des proportions démesurées en exerçant des effets négatifs sur la population; malgré la prévention et les traitements, le nombre de nouveaux consommateurs d'héroïne, de décès dus à la toxicomanie et de personnes infectées par le VIH ne cessait de croître. La criminalité due à la drogue augmentait en parallèle. Les personnes

Figure 1

Réduction de la délinquance pendant le traitement à base d'héroïne (données de la police, $\mathrm{n}=604$, Killias et al. [1]).

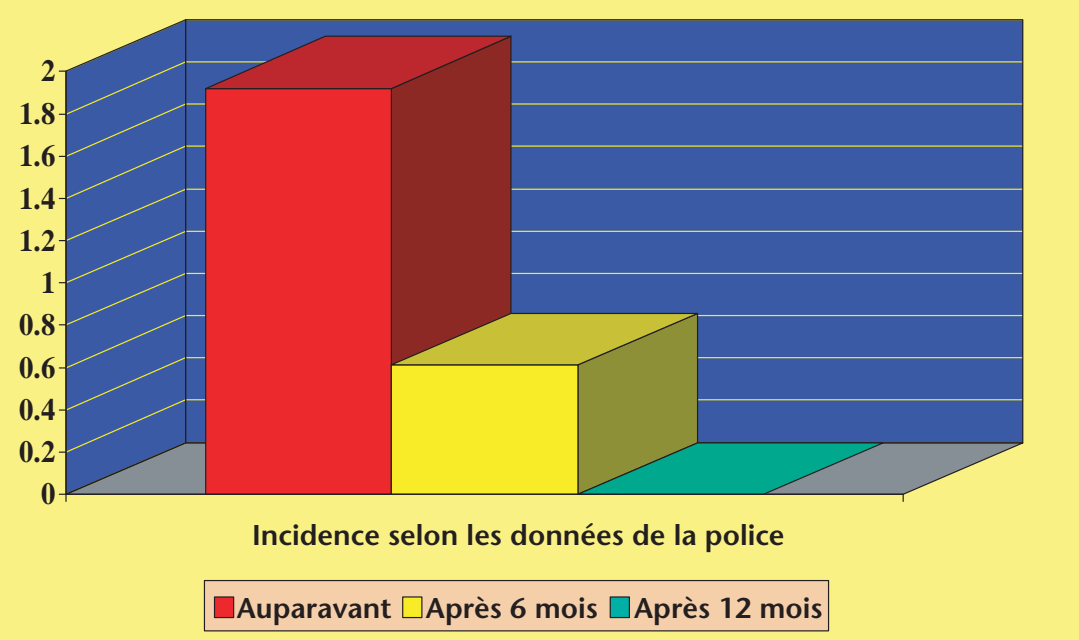

chargées d'intervenir ne disposaient d'aucune base d'action commune, que ce soit au niveau communal, cantonal ou fédéral, ni de perspective de résultats positifs.

La politique des quatre piliers a enfin apporté la base nécessaire pour

- une collaboration entre les intervenants et entre les niveaux d'intervention;

- de nouvelles initiatives dans la prévention (par exemple, prévention secondaire auprès de groupes-cibles particulièrement menacés);

- l'extension et la diversification des options thérapeutiques; ainsi que pour de nouvelles initiatives à bas seuil d'accès et pour le traitement des personnes qui avaient échappé à toute prise en charge ( $\mathrm{y}$ compris le traitement avec prescription d'héroïne);

- une réorientation des mesures répressives (tolérance zéro pour la consommation et le trafic de drogue dans l'espace public, ouverture de centres d'accueil et de transit en collaboration entre médecins et police, tolérance de la consommation non publique sans répercussions pour la population);

- la reconnaissance et la promotion de mesures de réduction des risques (prévention du sida par la distribution de matériel d'injection stérile, locaux d'injection, mesures réduisant les risques dans l'exécution des peines).

Les conséquences positives de cette politique apparaissent entre autres clairement dans les constats suivants:

- réduction du nombre de nouveaux consommateurs d'héroïne;

- réduction des décès dus à la toxicomanie;

- réduction du nombre d'héroïnomanes non pris en charge;

- réduction des comportements à risque et d'infections VIH chez les toxicomanes; 
- réduction de la délinquance due à la drogue dans les villes;

- disparition des scènes ouvertes insensées et de leurs conséquences;

- conséquences sociales et médicales largement positives et durables chez les patients bénéficiant de la prescription d'héroïne.

Par contre, les conséquences négatives redoutées par les opposants à cette nouvelle politique ne sont pas survenues:

- pas d'augmentation de l'attrait de l'héroïne pour les adolescents;

Figure 2

Nouveaux consommateurs d'héroïne (taux d'incidence Nordt und Stohler [2]).

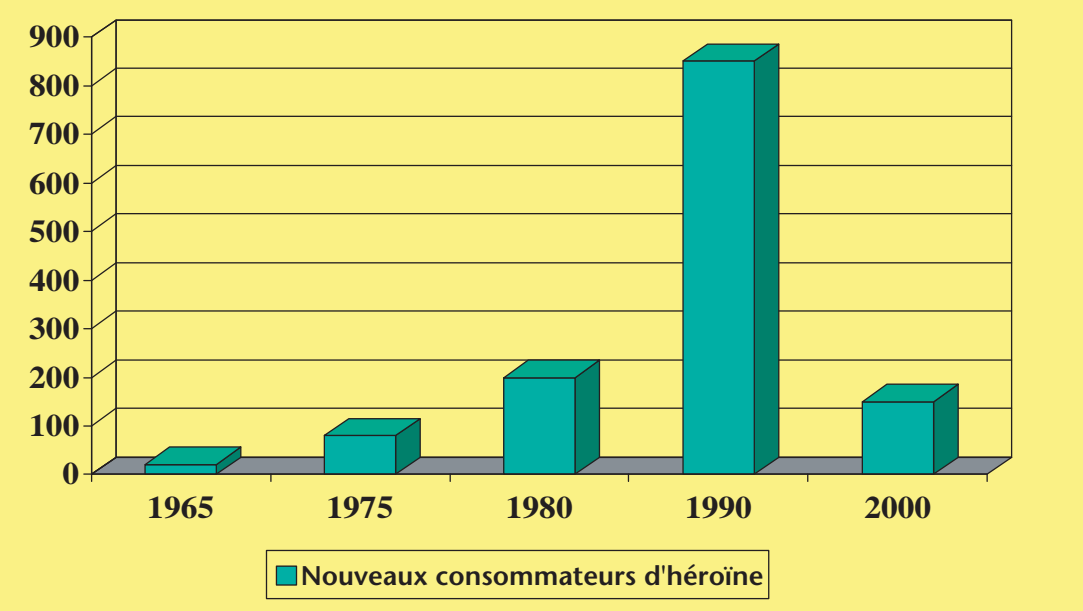

- pas de maintien dans la dépendance à l'héroïne suite au traitement avec prescription d'héroïne;

- pas de rejet des autres formes thérapeutiques (p.ex. axées sur l'abstinence) suite à la disponibilité de traitements avec prescription d'héroïne.

Après un début marqué par le scepticisme, les succès obtenus par cette politique des quatre piliers sont reconnus sur le plan européen et ont fortement marqué la politique de la drogue et la pratique en la matière.

Cette politique est-elle encore nécessaire? La population continue d'être sujette aux risques d'addiction. D'autres drogues que l'héroïne ont passé au premier plan (cocaïne, drogues synthétiques, alcool, médicaments), et les problèmes de la toxicomanie ont disparu des rues et de la conscience publique. La poursuite des mesures appliquées depuis 1991 reste malgré cela impérative si l'on veut éviter un retour de la situation dramatique antérieure.

\section{Références}

1 Killias M, Aebi MF, Ribaud D. Effects of heroin prescription on police contacts among drug addicts. Eur J Crim Policy Res. 1998;6:433-8.

2 Nordt C, Stohler R. Incidence of heroin use in Zurich, Switzerland: a treatment case register analysis. Lancet. 2006;367(9525):1830-4. 